\section{ÄDA-Kongress Bonn}

\section{Seminar: \\ "Der allergische Schock“}

\section{Samstag, den 2. September, 8.30-11.30 und 14.45-17.45 Uhr}

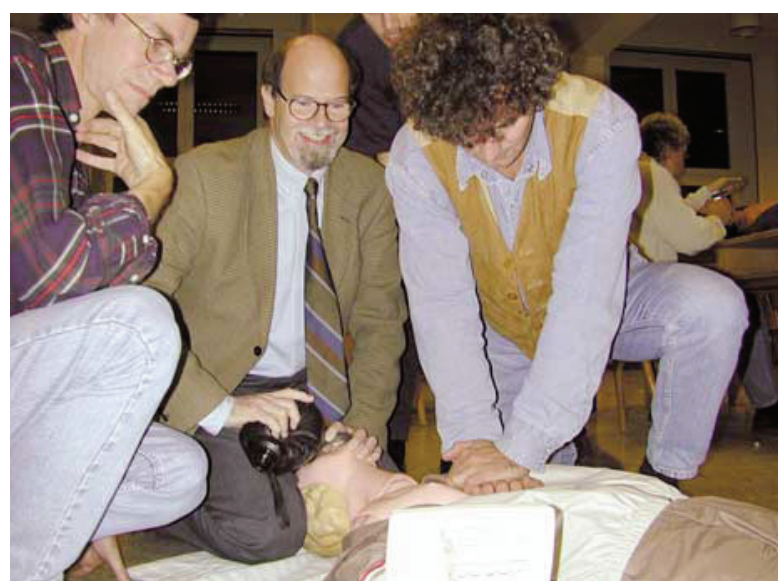

Durch Fortbildung adäquat auf Notfälle reagieren

D as plötzliche und meist unerwartete Auftreten von schweren anaphylaktischen und anaphylaktoiden Schockreaktionen - gar nicht so selten lebensbedrohend - erfordert eine adäquate Notfallbehandlung. Diese kann nur erfolgreich sein, wenn die notwendigen Maßnahmen so schnell und zielgerecht wie möglich durchgeführt werden. Ärzte nahezu aller Disziplinen

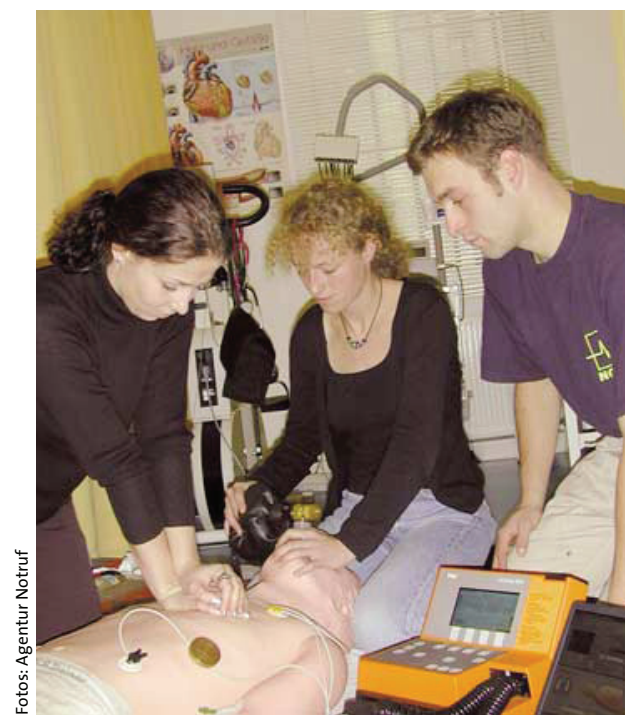

können mit einer derartigen Problematik konfrontiert werden - sei es im ärztlichen Notdienst - sei es in eigener Praxis bei der Injektion von z.B. Antibiotika, Antipyretika oder Hyposensibilisierungslösungen.

Nur der ausreichend ausgebildete Arzt wird in der Lage sein, Erste-Hilfe-Maßnahmen inklusive einer angepassten medikamentösen Behandlung durchzuführen.

Die Berufsordnung und die Weiterbildungsordnung der Heilberufe verlan-

\begin{tabular}{|l|}
\hline Programm und Anmeldung \\
- Univ.-Prof. Dr. med. Günther Forck \\
Medikamentöse Therapie \\
Dr. med. Marcus Schubert \\
Reanimation mit Training \\
Matthias Kühn und Mitarbeiter \\
der Agentur ,Notruf“, Berlin \\
Praktische Übungen \\
Da die Teilnehmerzahl vormittags und \\
nachmittags nur je 30 Personen zu- \\
lässt, entscheidet das Datum der \\
Anmeldung über die Teilnahme. \\
Anmeldung bei der ÄDA-Geschäfts- \\
stelle Blumenstr. 14 in 63303 Dreieich, \\
Tel. $06103-63657$, Fax -67674 \\
Kongress-Konto 506606032 \\
BLZ 50592200, Volksbank Dreieich \\
\hline
\end{tabular}

gen derartige Kenntnisse. Nutzen Sie diesen Intensivkurs, um sich mit praktischen Übungen für die Notfallsituation fit zu machen. Ein Team von Ärzten, Helfern und Phantomen wird ein äußerst realistisch konzipiertes Szenarium bieten.

Machen Sie mit!

G. Forck, Münster ( $\ddot{A} D A)$, Agentur Notfall, Berlin

\title{
Klaus Kalveram-Stiftung, Münster
}

\section{Der Klaus Kalveram-Preis wird zum 3. Mal verliehen}

$\mathrm{D}$ er mit 5.000 DM dotierte Klaus Kalveram-Preis soll anlässlich des 26. ÄDA-Kongresses vom 1.-3. September in Bonn zum dritten Mal verliehen werden. Der 1995 gestiftete Preis dient der Förderung von Nachwuchswissenschaftlern in der Allergologie, speziell im Bereich der Arzneimittelund Nahrungsmittelallergien.

Berücksichtigt werden abgeschlossene, wissenschaftliche Arbeiten zum Thema Arznei- und Nahrungsmittelallergien. Sie können bereits publiziert, sollten dann aber nicht älter als zwei Jahre sein.

Die Bewerber werden gebeten, ihre Arbeiten in deutscher oder englischer
Sprache bis zum 1. August 2000 beim Vorsitzenden der Klaus Kalveram-Stiftung,

Herrn Prof. Dr. Günther Forck

Kirchherrngasse 14

48143 Münster

in dreifacher Ausführung einzureichen. Eventuelle Anfragen sind ebenfalls an diese Adresse zu richten.

Die Arbeit muss ebenso wie das beigefügte Autorenreferat ohne Namensvermerk oder Hinweis auf den Autor sein. Name und Anschrift des Bewerbers bitte in einem verschlossenen Umschlag dem Manuskript beifügen.

G. Forck, Münster 